\title{
The variational multiscale element free Galerkin method for the simulation of power-law fluid flows
}

\author{
Xiaohua Zhang \& Ping Zhang \\ College of Science, China Three Gorges University, China
}

\begin{abstract}
In this work, the variational multiscale element free Galerkin method is used for the solution of incompressible generalized Newtonian fluid flow. In order to correct the lack of stability of the standard Galerkin formulation of the NavierStokes equations, the velocity field is decomposed into coarse and fine scales first, and then a model for the fine scale velocity is introduced, in the process, the stabilization parameter has appeared naturally via the solution of the fine scale problem. From the viewpoint of the application, the presented method can employ an equal order basis for pressure and velocity that is easy to implement but avoid the restriction of the Babuska-Brezzi condition. Two benchmark problems named Poiseuille flow and lid-driven cavity flow for the power-law are solved and the numerical results confirm that this method has better stability and accuracy.

Keywords: variational multiscale, generalized Newtonian fluid, power-law fluid, element free Galerkin method.
\end{abstract}

\section{Introduction}

Meshless methods are very efficient for solving the practical engineering problems because they rely only on a set of scattered nodes in the problem domain, which can not only enable them to eliminate at least part of the difficulties existing in FEM, but also describe more accurately the irregular geometries. A detailed review on the meshless methods has been provided by Nguyen et al. [1] and Liu and Gu [2]. Recently many meshless methods have been proposed and applied to computational fluid dynamics [3-14]. 
It is well known that the one of main issues germane to the development of a successful solver for incompressible Navier-Stokes equations is proper treatment of incompressibility. Improper treatments may result in spurious oscillations for velocity or pressure solutions. For meshless methods based on Galerkin formulations, the same problems will arise from the treatment of incompressiblility constraint $[6,8]$, that is, they must be either satisfy or avoid the Babuska-Brezzi condition. In order to eliminate numerical instability due to the improper coupling of velocity and pressure, some stabilized techniques which frequently used in the finite element context were directly extended to meshless method [3-9]. However, these methods are related to a stabilization parameter and it depends on the problem under consideration and the chosen numerical method. In other words, there was no effective theoretical framework to solve the stabilized factor or stabilized functions which are closely connecting with the accuracy and reliability for numerical method. In order to overcome this problem, Zhang et al. developed variational multiscale element free Galerkin (VMEFG) method for the solution of Stokes problem [10]. VMEFG method inherits the advantages of the VMFEM, that is, it allows equal order basis for pressure and velocity and the stabilized parameter appear naturally. Subsequently, Zhang et al. applied VMEFG to simulate the water wave problems [11] and MHD flow problems [12]. To the best of our knowledge, there are few published results when meshless method is used to non-Newtonian fluid flow problems and meshless method for the simulation of non-Newtonian are usually applied in collocation sets $[13,14]$. Although meshless collocation methods do not need background quadrature mesh and make the computation efficient, they may suffer from the accuracy and stability problems. Therefore, in the present study VMEFG method is extended to the solution of incompressible generalized Newtonian fluid flow.

An outline of the paper is as follow. In section 2, a brief description of the rheological properties of power-law fluids is given. In section 3, the fundamental principle of EFG method is briefly described. Then, the VMEFG method for the Stokes fluid flow is expressed. In section 5, Poiseuille flow and lid-driven cavity flow of a power-law fluid at low Reynolds numbers are solved as two benchmark problems and the results are compared with analytical solutions or the available numerical data. The article ends with concluding remarks in section 6 .

\section{Governing equations}

\subsection{Conservation equations}

The governing equations for simulating the two-dimensional incompressible flow of generalized Newtonian fluid are written in a dimensionless form as

$$
\begin{gathered}
\nabla \cdot \boldsymbol{u}=0 \\
\frac{\partial \boldsymbol{u}}{\partial t}+\boldsymbol{u} \cdot \nabla \boldsymbol{u}-\frac{1}{\operatorname{Re}} \nabla \cdot \boldsymbol{\tau}+\nabla p=\boldsymbol{F}
\end{gathered}
$$

where $\boldsymbol{u}=(u, v)^{\mathrm{T}}$ is the fluid velocity, $p$ is the pressure, $\boldsymbol{F}$ is body force that drives fluid and $\tau$ is shear stress tensor, Re is the Reynolds number. The above 
equations are written in general form and are applicable to both Newtonian and non-Newtonian flows.

\subsection{Rheology}

For engineering applications, the power-law model is commonly used for modelling shear-thinning or shear-thickening behaviour of non-Newtonian fluids. The shear-stress tensor in the power-law is expressed by

$$
\tau_{i j}=2 \eta \gamma_{i j}
$$

where $\boldsymbol{\gamma}_{i j}=\frac{1}{2}\left(\frac{\partial \boldsymbol{u}_{i}}{\partial \boldsymbol{x}_{j}}+\frac{\partial \boldsymbol{u}_{j}}{\partial \boldsymbol{x}_{i}}\right)$ with $\boldsymbol{u}_{i}$ being the velocity component in $\boldsymbol{x}_{i}$ direction, $\eta$ is the generalized Newtonian viscosity which depends on the local shear rate. In Newtonian fluid $\eta$ is constant for the entire flow field and for power-law model non-Newtonian viscosity is given by

$$
\eta=\mu_{0} \dot{\gamma}^{n-1}
$$

where $\mu_{0}$ a constant relative to the properties of the fluid and $n$ is the exponent according to which shear thinning or shear thickening behavior is regarded when $n<1$ or $n>1$, respectively. In the special case of $n=1$, the model reduces to the one-parameter Newtonian case. The strain-rate tensor is obtained by

$$
\dot{\gamma}=\sqrt{2 \gamma_{i j} \gamma_{i j}}
$$

Using constitutive equations, Eq. (2) will be

$$
\frac{\partial \boldsymbol{u}}{\partial t}+\boldsymbol{u} \cdot \nabla \boldsymbol{u}-\frac{1}{\operatorname{Re}} \nabla \cdot(\eta \nabla \boldsymbol{u})+\nabla p=\boldsymbol{F}
$$

\section{Review of the element free Galerkin method}

In the EFG method, the field variable $u(\boldsymbol{x})$ is approximated by moving least squares (MLS) approximation, which consists of three parts: a basis function, a group of nonconstant coefficients and a weight function associated with each node. According to the MLS approximation, the unknown function $u(x)$ can be written as follows [2]:

$$
u(\boldsymbol{x}) \approx u^{h}(\boldsymbol{x})=\sum_{j=1}^{m} p_{j}(\boldsymbol{x}) a_{j}(\boldsymbol{x})=\boldsymbol{P}^{\mathrm{T}}(\boldsymbol{x}) \boldsymbol{a}(\boldsymbol{x})
$$

where $\boldsymbol{P}(\boldsymbol{x})$ is a complete polynomial basis of order $m$ and $\boldsymbol{a}(\boldsymbol{x})$ is a vector of coefficients (to be determined) which is a function of the space coordinate $\boldsymbol{x}$.

Assume that we have known the nodal value $u_{i}=u\left(\boldsymbol{x}_{i}\right)$ for the function $u(\boldsymbol{x})$ at $n$ nodes in the domain $\Omega$. Then the unknown coefficients $\boldsymbol{a}(\boldsymbol{x})$ in Eq. (7) at any given point $\boldsymbol{x}$ are determined by minimizing the functional $\boldsymbol{J}$ :

$$
\boldsymbol{J}=\sum_{i=1}^{n} w\left(\boldsymbol{x}-\boldsymbol{x}_{i}\right)\left[u_{i}-\boldsymbol{P}^{\mathrm{T}}\left(\boldsymbol{x}_{i}\right) \boldsymbol{a}(\boldsymbol{x})\right]^{2}
$$


Here $n$ is the number of nodes in the support domain of $\boldsymbol{x}$ in which the weigh function $w\left(\boldsymbol{x}-\boldsymbol{x}_{i}\right)>0$. The cubic spline weight function has been used in this work, which is given as:

$$
w(\bar{d})=\left\{\begin{array}{cc}
\frac{2}{3}-4 \bar{d}^{2}+4 \bar{d}^{3} & 0 \leq \bar{d} \leq 0.5 \\
\frac{4}{3}-4 \bar{d}+4 \bar{d}^{2}-\frac{4}{3} \bar{d}^{3} & 0.5<\bar{d} \leq 1 \\
0 & \text { otherwise }
\end{array}\right.
$$

where $\bar{d}=d_{i} / r_{i}, d_{i}=\left\|\boldsymbol{x}-\boldsymbol{x}_{i}\right\|$ is the distance between point $\boldsymbol{x}$ and node $\boldsymbol{x}_{i}, r_{i}$ is the radius of the influence domain for node $\boldsymbol{x}_{i}$. Generally, $r_{i}=\alpha \rho_{i}$ where $\alpha$ is the dimensionless size of influence domain and $\rho_{i}$ is the distance between two adjacent nodes.

Minimization of Eq. (8) with respect to $\boldsymbol{a}(\boldsymbol{x})$ then yields the following system of linear equations for the vector $\boldsymbol{a}(\boldsymbol{x})$ :

$$
A(x) a(x)=B(x) u
$$

where $\boldsymbol{u}$ is the vector of nodal unknowns, matrices $\boldsymbol{A}(\boldsymbol{x})$ and $\boldsymbol{B}(\boldsymbol{x})$ are defined as

$$
\begin{gathered}
\boldsymbol{A}(\boldsymbol{x})=\sum_{i=1}^{n} w_{i}(\boldsymbol{x}) \boldsymbol{P}\left(\boldsymbol{x}_{i}\right) \boldsymbol{P}^{\mathrm{T}}\left(\boldsymbol{x}_{i}\right) \\
\boldsymbol{B}(\boldsymbol{x})=\left[w_{1}(\boldsymbol{x}) \boldsymbol{P}\left(\boldsymbol{x}_{1}\right), w_{2}(\boldsymbol{x}) \boldsymbol{P}\left(\boldsymbol{x}_{2}\right), \cdots, w_{n}(\boldsymbol{x}) \boldsymbol{P}\left(\boldsymbol{x}_{n}\right)\right]
\end{gathered}
$$

If $\boldsymbol{A}$ is invertible, solving Eq. (10) and then substituting $\boldsymbol{a}(\boldsymbol{x})$ back into Eq. (7), the MLS approximant can be defined as

$$
u^{h}(\boldsymbol{x})=\boldsymbol{P}^{\mathrm{T}}(\boldsymbol{x}) \boldsymbol{A}^{-1}(\boldsymbol{x}) \boldsymbol{B}(\boldsymbol{x}) \boldsymbol{u}=\boldsymbol{N}^{\mathrm{T}}(\boldsymbol{x}) \boldsymbol{u}
$$

where $N(x)$ is the vector of MLS shape functions. Please refer to the book of Liu and $\mathrm{Gu}[2]$ in detail.

In general, the use of the MLS approximation produces shape functions that do not possess the Kronecker Delta condition property, i.e., $\boldsymbol{N}_{i}\left(\boldsymbol{x}_{j}\right) \neq \delta_{i j}$. Thus, essential boundary conditions cannot be imposed as easily as that in the standard FEM. Until now, the implementation of essential boundary conditions is still an open research topic for meshless methods. In the paper, a simple technique proposed by Zhang et al. $[11,12]$ is utilized, which makes MLS approximation function possess interpolation property as $\alpha$ approach to 1 and is easier to solve problems with complex area.

\section{The variational multiscale element free Galerkin method}

The VMEFG method essentially contains three steps for the simulation of incompressible fluid flow. In the first step, the velocity field is decomposed into coarse scale and fine scale. In the second step, it uses bubble function to determine fine scale solution analytically. In the third step, substitutes the fine scale solution into coarse scale problem and then obtains the coarse scale solution numerically. In the following, the brief introduction of VMEFG is presented and more details about the VMEFG can refer to $[10,11]$. 


\subsection{The standard weak form}

Let $\boldsymbol{w}$ and $q$ represent the weight functions for velocity $\boldsymbol{u}$ and pressure $p$ respectively, then based on the Galerkin formulation, the standard weak form of the problem Eqs. (1) and (6) is given as follows

$$
\begin{gathered}
(\boldsymbol{w}, \boldsymbol{u} \cdot \nabla \boldsymbol{u})+\frac{1}{\operatorname{Re}}(\nabla \boldsymbol{w}, \eta \nabla \boldsymbol{u})-(\nabla \cdot \boldsymbol{w}, p)=(\boldsymbol{w}, \boldsymbol{F}) \\
(q, \nabla \cdot \boldsymbol{u})=0
\end{gathered}
$$

where $(\cdot, \cdot)=\int_{\Omega}(\cdot) d \Omega$.

\subsection{The decomposition of the standard weak form}

Assume that the velocity $\boldsymbol{u}$ and its weight function $\boldsymbol{w}$ can be decomposed into coarse scale and fine scale respectively, namely $\boldsymbol{u}=\overline{\boldsymbol{u}}+\hat{\boldsymbol{u}}$ and $\boldsymbol{w}=\overline{\boldsymbol{w}}+\hat{\boldsymbol{w}}$, where $\overline{\boldsymbol{u}}$ and $\hat{\boldsymbol{u}}$ are the coarse scale and fine scale for velocity $\boldsymbol{u}, \overline{\boldsymbol{w}}$ and $\hat{\boldsymbol{w}}$ are the coarse scale and fine scale for weight function $\boldsymbol{w}$, respectively. Meanwhile, we assume there exists a linearity between coarse scale and fine scale [10]. We further assume that $\hat{\boldsymbol{u}}$ and $\hat{\boldsymbol{w}}$ although non-zero within background integral cell $\Omega^{K}$, vanish identically over the $\Omega^{K}$ boundaries when influence factor $\alpha$ approaches to 1 .

Substituting $\boldsymbol{u}=\overline{\boldsymbol{u}}+\hat{\boldsymbol{u}}$ and $\boldsymbol{w}=\overline{\boldsymbol{w}}+\hat{\boldsymbol{w}}$ into the standard variational form Eqs. (14)-(15), then employing the linearity between coarse scale $\overline{\boldsymbol{w}}$ and fine scale $\hat{\boldsymbol{w}}$, meanwhile using the converged solution $\boldsymbol{u}^{c}$ at the last time to linearize the nonlinear convective coefficient, Eq. (14) can be split into coarse scale problem $\bar{W}$ and fine scale problem $\hat{W}$ as follows:

$$
\begin{aligned}
& \left(\overline{\boldsymbol{w}}, \boldsymbol{u}^{c} \cdot \nabla(\overline{\boldsymbol{u}}+\hat{\boldsymbol{u}})+\frac{1}{\operatorname{Re}}(\nabla \overline{\boldsymbol{w}}, \eta \nabla(\overline{\boldsymbol{u}}+\hat{\boldsymbol{u}}))-(\nabla \cdot \overline{\boldsymbol{w}}, p)=(\overline{\boldsymbol{w}}, \boldsymbol{F})\right. \\
& \left(\hat{\boldsymbol{w}}, \boldsymbol{u}^{c} \cdot \nabla(\overline{\boldsymbol{u}}+\hat{\boldsymbol{u}})\right)+\frac{1}{\operatorname{Re}}(\nabla \hat{\boldsymbol{w}}, \eta \nabla(\overline{\boldsymbol{u}}+\hat{\boldsymbol{u}}))-(\nabla \cdot \hat{\boldsymbol{w}}, p)=(\hat{\boldsymbol{w}}, \boldsymbol{F})
\end{aligned}
$$

\subsection{The solution of the fine scale problem}

In general, according to [10], we solve the fine scale problem Eq. (17) first to obtain the fine scale solution $\hat{\boldsymbol{u}}$. In order to obtain fine scale solution $\hat{\boldsymbol{u}}$ analytically from $\hat{W}$, using the bubble function in each $\Omega^{K}$, and finally we can obtain the fine scale solution $\hat{\boldsymbol{u}}$ over the $\Omega^{K}$ as follows:

$$
\left.\hat{\boldsymbol{u}}\right|_{\Omega^{k}}=b_{1}^{K}(\boldsymbol{\xi}) \boldsymbol{K}^{-1} \boldsymbol{R}
$$

where

$$
\begin{gathered}
\boldsymbol{K}=\int_{\Omega^{k}} b_{2}^{K} \boldsymbol{u}^{c} \cdot \nabla b_{1}^{K} d \Omega \boldsymbol{I}+\frac{1}{\operatorname{Re}}\left(\int_{\Omega^{k}} \eta \nabla b_{1}^{K}: \nabla b_{2}^{K} d \Omega \boldsymbol{I}+\int_{\Omega^{k}} \eta \nabla b_{1}^{K} \otimes \nabla b_{2}^{K} d \Omega\right)( \\
\boldsymbol{R}=\int_{\Omega^{k}} b_{2}^{K} \overline{\boldsymbol{r}} d \Omega
\end{gathered}
$$




$$
\overline{\boldsymbol{r}}=\boldsymbol{F}-\boldsymbol{u}^{c} \cdot \nabla \overline{\boldsymbol{u}}+\frac{1}{\mathrm{Re}} \nabla \cdot(\eta \nabla \overline{\boldsymbol{u}})-\nabla p
$$

Here, $\boldsymbol{I}$ is a $n d \times n d$ identity matrix, both $\nabla b_{1}^{K}$ and $\nabla b_{2}^{K}$ are $n d \times 1$ vectors of gradient of the bubble function, $n d$ indicates the dimension of the problem.

\subsection{The solution of the coarse scale problem}

Once we have obtained the fine scale solution $\hat{\boldsymbol{u}}$, substitute it into the coarse fine problem. We get

$$
\begin{aligned}
&\left(\overline{\boldsymbol{w}}, \boldsymbol{u}^{c}\right.\cdot \nabla \overline{\boldsymbol{u}})+\frac{1}{\operatorname{Re}}(\nabla \overline{\boldsymbol{w}}, \eta \nabla \overline{\boldsymbol{u}})-(\nabla \cdot \overline{\boldsymbol{w}}, p) \\
&+\left(\boldsymbol{u}^{c} \cdot \nabla \overline{\boldsymbol{w}}+\frac{1}{\operatorname{Re}} \nabla \cdot(\eta \nabla \overline{\boldsymbol{w}}), \boldsymbol{\kappa}\left(\boldsymbol{u}^{c} \cdot \nabla \overline{\boldsymbol{u}}-\frac{1}{\mathrm{Re}} \nabla \cdot(\eta \nabla \overline{\boldsymbol{u}})+\nabla p\right)\right) \\
&=(\overline{\boldsymbol{w}}, \boldsymbol{F})+\left(\boldsymbol{u}^{c} \cdot \nabla \overline{\boldsymbol{w}}+\frac{1}{\operatorname{Re}} \nabla \cdot(\eta \nabla \overline{\boldsymbol{w}}), \boldsymbol{\kappa} \boldsymbol{F}\right) \\
&(q, \nabla \cdot \overline{\boldsymbol{u}})+\left(\nabla q, \boldsymbol{\kappa}\left(\boldsymbol{u}^{c} \cdot \nabla \overline{\boldsymbol{u}}-\frac{1}{\mathrm{Re}} \nabla \cdot(\eta \nabla \overline{\boldsymbol{u}})+\nabla p\right)\right)=(\nabla q, \boldsymbol{\kappa} \boldsymbol{F})
\end{aligned}
$$

where

$$
\boldsymbol{\kappa}=b_{1}^{K} \int_{\Omega^{k}} b_{2}^{K} d \Omega\left[\begin{array}{l}
\int_{\Omega^{k}} b_{2}^{K} \boldsymbol{u}^{c} \cdot \nabla b_{1}^{K} d \Omega \boldsymbol{I} \\
+\frac{1}{\operatorname{Re}}\left(\int_{\Omega^{k}} \eta \nabla b_{1}^{K}: \nabla b_{2}^{K} d \Omega \boldsymbol{I}+\int_{\Omega^{k}} \eta \nabla b_{1}^{K} \otimes \nabla b_{2}^{K} d \Omega\right)
\end{array}\right]^{-1}
$$

Compared with the standard variational form, it is important to note that the fourth term on the left appears in Eq. (22) and the second term on the left appears in Eq. (23) owe to the assumption that there exists fine scale in the problem, which in fact models the effect of the fine scale. Additionally, the method is based on residual, therefore the resulting formulation is consistent and can accommodate the exact solution. Another important feature of this formulation is that the definition of the stabilization tensor $\boldsymbol{\kappa}$ has appeared naturally via the solution of the fine scale problem.

\section{Numerical example}

In this section, two benchmark problems named plane Poiseuille flow and liddriven cavity flow are solved to demonstrate the performance of the VMEFG method is solving non-Newtonian flows.

\subsection{Plane Poiseuille flow}

The Poiseuille flow is formed between two parallel plates. A schematic of the problem is depicted in Fig. 1. The width of the channel is 2 units and the length of the channel is 6 units. No-slip conditions are imposed on the solid boundaries. Results of numerical simulation using $p$-version least squares finite element 
method were presented by Bell and Surana [15] and smoothed particle hydrodynamics were presented by Vakilha and Manzari [13]. The analytical solution of the velocity profile for a fully-developed laminar flow of a power-law fluid is given by

$$
\frac{v(y)}{v_{\text {avg }}}=\frac{2 n+1}{n+1}\left[1-\left(\frac{2|y|}{L}\right)^{\frac{n+1}{n}}\right]
$$

where $v$ is normalized by the average velocity $v_{\text {avg }}$.

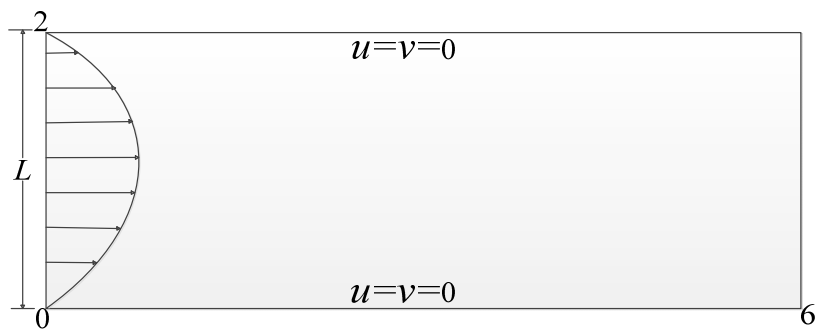

Figure 1: Geometry and boundary conditions for Poiseuille flow under parabolic inlet velocity profile.

Fig. 2 plots the full developed velocity profiles for power-law indices of 0.5 , 1.0 and 1.5 respectively. It can be noted that our calculated velocity profiles are in excellent agreement with the analytical solution.

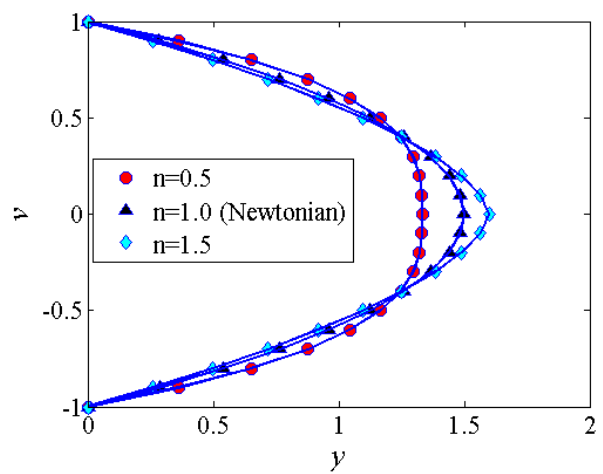

Figure 2: Velocity profiles for various values of the power-law index. Lines represent the analytical solution for various values of the powerlaw index.

\subsection{Lid-driven cavity flow}

Lid-driven cavity flow problem is also usually considered as a benchmark problem to validate the proposed methods for numerical simulations of nonNewtonian flow. The problem is characterized by a square cavity in which the 
driving force for the flow is the shear created by the sliding lid. Fig. 3 shows a schematic of the cavity with the boundary conditions.

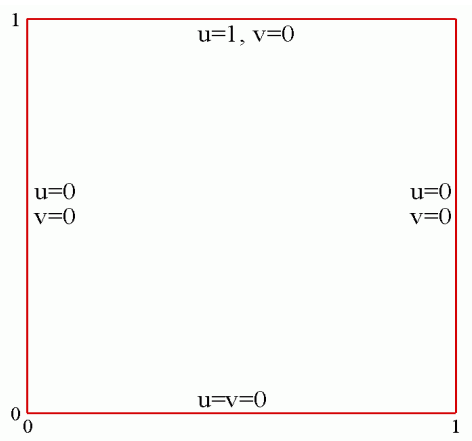

Figure 3: Geometry and boundary conditions for lid-driven cavity flow problem.

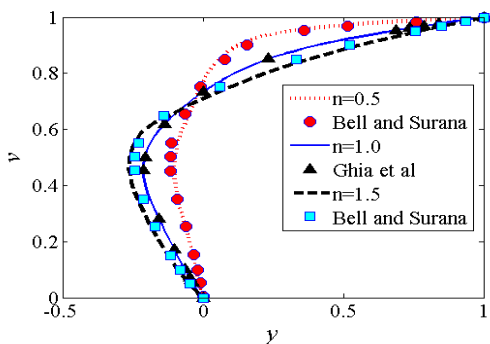

(a) $u$-velocity

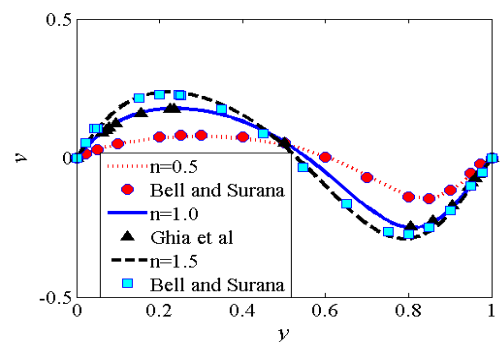

(b) $v$-velocity

Figure 4: Comparison between present results and the results by previous researchers for different values of power-law indices.

In order to validate the VMEFG method for the problem, calculations have been performed for $\mathrm{Re}=100$ and different values of the power-law indices of which results from previous research are available [15]. Figure 4 shows the present results for the $u$-velocity along the vertical centerline of the cavity and $v$ velocity along the horizontal centerline of the cavity for $n=0.5,1.0$ and 1.5 . It can be seen that for $n=0.5$ and 1.5 the results obtained by VMEFG are in good agreement with the results by Bell and Surana [15], and for $n=1.0$, these are in close with the results by Ghia et al. [16]. Figure 5 shows the streamline for power-law indices of $0.5,1.0$ and 1.5 respectively. It can be seen that the position of the main vortex shifts towards the upper right corner as the powerlaw index decreases. The pressure contours for power-law indices of $0.5,1.0$ and 1.5 are also shown in Figure 6, it can be seen that the pressure contours are smooth. 


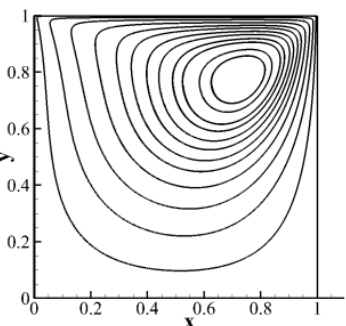

(a) $n=0.5$

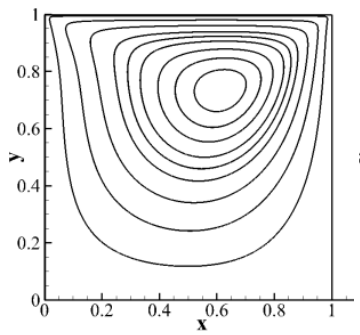

(b) $n=1.0$

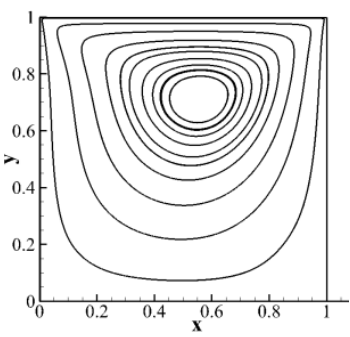

(c) $n=1.5$

Figure 5: $\quad$ Streamlines for power-law indices $n=0.5$ (a), $n=1.0$ (b), $n=1.5$ (c).

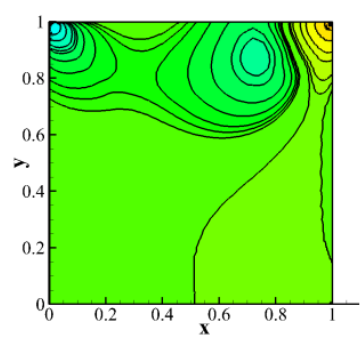

(a) $n=0.5$

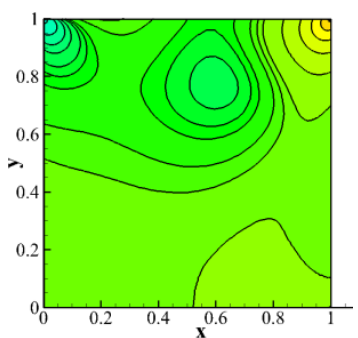

(b) $n=1.0$

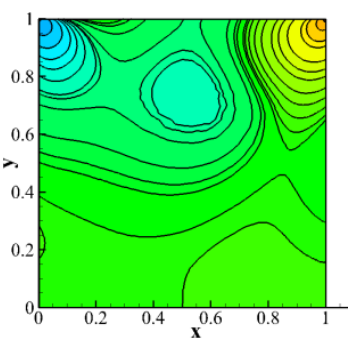

(c) $n=1.5$

Figure 6: $\quad$ Pressure contours for power-law indices $n=0.5$ (a), $n=1.0$ (b), $n=1.5$ (c).

\section{Conclusions}

In the work EFG combined with the variational multiscale method was extended for the solution of incompressible generalized Newtonian fluid flow. Numerical example indicates that VMEFG method can produce satisfactory numerical results for the solution of incompressible generalized Newtonian fluid flow in the simulation of laminar flow of power-law fluids. Additionally, although in the paper the presented formulation has been used for simulation of the laminar of power-law fluids, it also can be used for other generalized Newtonian model, such as the Carreau-Yasuda model, Bingham model and so on.

\section{Acknowledgements}

The support from the Natural Sciences Foundation of China (No. 11102101), the Academic Mainstay Foundation of Hubei Province of China (No. T201103), the Science Foundation of China Three Gorges University (No. KJ2009B058) are fully acknowledged. 


\section{References}

[1] V. P. Nguyen, T. Rabczuk, S. Bordas, M. Duflot. Meshless methods: a review and computer implementation aspects. Mathematics and Computers in Simulation, 2008, 79: 763-813.

[2] G. R. Liu, Y. T. Gu. An Introduction to Meshfree Methods and their Programming. Springer: Netherlands, 2005.

[3] T. P. Fries, H. G. Matthies. A stabilized and coupled meshfree/meshbased method for the incompressible Navier-Stokes equations - Part I: Stabilization. Computer Methods in Applied Mechanics and Engineering, 2006, 195(44-47): 6205-6224.

[4] T. P. Fries, H. G. Matthies. A stabilized and coupled meshfree/meshbased method for the incompressible Navier-Stokes equations-Part II: Coupling. Computer Methods in Applied Mechanics and Engineering, 2006, 195(4447): 6191-6204.

[5] X. K. Li, Q. L. Duan. Meshfree iterative stabilized Taylor-Galerkin and Characteristic-Based Split (CBS) algorithms for incompressible N-S equations. Computer Methods in Applied Mechanics and Engineering, 2006, 195(44-47): 6125-6145.

[6] A. Huerta, Y. Vidal, P. Villon. Pseudo-divergence-free element free Galerkin method for incompressible fluid flow. Computer Methods in Applied Mechanics and Engineering, 2004, 193(12-14): 1119-1136.

[7] H. Lin, S. N. Atluri. The meshless local Petrov-Galerkin (MLPG) method for solving incompressible Navier-Stokes equations. Comput. Model. Eng. Sci., 2001, 2: 117-14.

[8] Q. L. Duan, X. K. Li. A meshless PSPG formulation for the incompressible Stokes flow. Chinese Journal of Computational Mechanics, 2007, 24: 192196.

[9] X. K. Zhang, K. C. Kwon, S. K. Youn. Least-squares meshfree method for incompressible Navier-Stokes problems. International Journal for Numerical Methods in Fluids, 2004, 46(3): 263-288.

[10] L. Zhang, J. Ouyang, X. H. Zhang. On a multi-scale element-free Galerkin method for the Stokes problem. Applied Mathematics and Computation, 2008, 203(2): 745-753.

[11] L. Zhang, J. Ouyang, T. Jiang, C. Ruan. Variational multiscale element free Galerkin method for the water wave problems. Journal of Computational Physics, 2011, 230: 5045-5060.

[12] L. Zhang, J. Ouyang, X. H. Zhang. The variational multiscale element free Galerkin method for MHD flows at high Hartmann numbers. Computer Physics Communications. 2013, 184: 1106-1118.

[13] M. Vakilha, M. T. Manzari. Modelling of power-law fluid flow through porous media using smoothed particle hydrodynamics. Transport in Porous Media, 2008, 74: 331-346.

[14] M. Lashcharbolok, E. Jabbari. Collocated discrete least squares (CDLS) meshless method for the simulation of power-law fluid flows. Scientia Iranica B, 2013, 20: 322-328. 
[15] B. C. Bell, K. S. Surana. p-version least squares finite element formulation for two-dimensional, incompressible, non-Newtonian isothermal and nonisothermal fluid flow. International Journal for Numerical Methods in Fluids, 1994, 18: 127-162.

[16] U. Ghia, K. N. Ghia, C. T. Shin. High-Re solutions for incompressible flow using the Navier-Stokes equations and a multigrid method. Journal of Computational Physics, 1982, 48: 387-411. 